\title{
Distributed Tracking Control for Discrete-Time Multiagent Systems with Novel Markovian Switching Topologies
}

\author{
Hongbin Wang, Zhen Zhou, and Zhongquan Hu \\ Key Lab of Industrial Computer Control Engineering of Hebei Province, Yanshan University, Qinhuangdao 066004, China \\ Correspondence should be addressed to Zhen Zhou; zhouzhen0617@163.com
}

Received 28 August 2017; Revised 27 November 2017; Accepted 5 December 2017; Published 27 December 2017

Academic Editor: Rigoberto Medina

Copyright (C) 2017 Hongbin Wang et al. This is an open access article distributed under the Creative Commons Attribution License, which permits unrestricted use, distribution, and reproduction in any medium, provided the original work is properly cited.

\begin{abstract}
Distributed discrete-time coordinated tracking control problem is investigated for multiagent systems in the ideal case, where agents with a fixed graph combine with a leader-following group, aiming to expand the function of the traditional one in some scenes. The modified union switching topology is derived from a set of Markov chains to the edges by introducing a novel mapping. The issue on how to guarantee all the agents tracking the leader is solved through a PD-like consensus algorithm. The available sampling period and the feasible control gain are calculated in terms of the trigonometric function theory, and the mean-square bound of tracking errors is provided finally. Simulation example is presented to demonstrate the validity of the theoretical results.
\end{abstract}

\section{Introduction}

Inspired by the potential applications in engineering, such as networked autonomous vehicles, sensor networks [1], and formation control [2], distributed coordination of multiagent systems has attracted much attention from researchers [3, 4]. Very recently, consensus problems have been studied extensively as well as references therein [5, 6]. Many methods have been developed to deal with the consensus problem like linear system theory [7], impulsive control [8], convex optimization method [9], and so on. Due to the complexity of network, the control problem of multiagent systems will be very challenging and difficult. Therefore, how to design the consensus control protocol for multiagent systems becomes a significant research focus.

In most cases, the connectivity of graph might be unfixed; it may deteriorate the system performance and even cause instability. Therefore, some of the existing results concentrate on the ideal case where multiagent systems can be described as the dynamic topology [10, 11]. Researching among those works, some main results and progress on distributed coordination control were given and the system under a dynamic topology was addressed through various methods $[12,13]$. For example, distributed consensus problem was studied for discrete-time multiagent systems with the switching graphs, where each agent's velocity was constrained to lie in a nonconvex set [14]. Moreover, two consensus problems were solved under a switching topology, which was assumed to be uniformly connected only [15]. Otherwise, the aforementioned works not only focused on first-order and second-order systems [16], but also focused on EulerLagrange models $[17,18]$ and even took the time delay and noises into account $[19,20]$.

Due to the random link failures, variation meeting the need and sudden environmental disturbances, some dynamical systems could be modeled as Markovian switching systems, which are starting with a rapid development [21-23]. Leader-following consensus problem was studied for data-sampled multiagent systems under the Markovian switching topologies [24] and a more interesting case with multiple dynamic leaders was considered in [25]. In [26], under a switching topology governed by Markov chains, the consensus seeking problem was solved through a guaranteed cost control method. It was unnecessary for the Markov chain to be ergodic, since each topology had a spanning tree. In addition, it is difficult to obtain all the elements of the transition rate matrix, or some of the elements are not necessary to guarantee the system stability. Markovian switching model with partially unknown transition rates was considered in [27], and any knowledge of the unknown 
elements was needed in the design procedure of finite time synchronization controller.

However, many practical systems can be addressed as a dynamic model, such as replacing the broken agents in the group and expanding the function on the basis of traditional one. The time varying reference can be tracked firmly in the original system, whether the union system could achieve consensus in case of combining some followers with a fixed graph. Besides, as far as we know, the usual case, in which Markov chain used in, is the modes of the topologies, since all of the subgraphs and the transition rate matrix should be known or partly known clearly. In contrast with that, Markov chains are applied to the edges of graph in this paper, so that the union system could be discovered through introducing a novel mapping, together with the distributed tracking problem for the union system; it is a valuable topic to be researched.

The main purpose of this paper is to establish the Markovian switching topologies for the union system with two subgraphs. Through a novel mapping, Markovian switching topologies are governed by a set of Markov chains to the edges of the graph. Hence, distributed coordinated tracking control problem is solved via a PD-like consensus algorithm adopted from [16]. Different from [16], a sufficient condition on the system stability is obtained based on trigonometric function theory. As shown in the forward reference, the tracking errors are ultimately bounded, which is partly determined by the bounded changing rate and the number of agents. Simulation result can more fully prove the effectiveness of the strategy.

The rest of this paper is organized as follows. In Section 2, graph theory based on a novel Markov process is given and PD-like consensus algorithm is adopted. In Section 3, stability analysis and some results are provided. Simulation example is presented in Section 4 and this paper is concluded in Section 5 .

\section{Preliminaries}

2.1. Graph Theory. Define a directed leader-following graph $G \triangleq(V, \varepsilon)$ with one leader labeled as node 0 and $n$ followers. $V=\left\{v_{0}, \ldots, v_{n}\right\}$ is a nonempty finite set of nodes and $\varepsilon \subseteq$ $V \times V$ is a set of edges. For an edge $\left(v_{i}, v_{j}\right) \in \varepsilon$, if the node $v_{j}$ can obtain information from $v_{i}, v_{i}$ is a neighbor of node $v_{j}$. A directed path is a sequence of edges in the form of $\left(v_{1}, v_{2}\right),\left(v_{2}, v_{3}\right),\left(v_{3}, v_{k}\right), \ldots$, where $v_{k} \in V$. The adjacency matrix $A=\left[a_{i j}\right] \in \mathbb{R}^{(n+1) \times(n+1)}$ is associated with $G$, where $a_{i j}>0$ if agent $v_{i}$ can obtain information from agent $v_{j}$ and $a_{i j}=0$ otherwise. Assume $a_{i i}=0$ and the leader does not receive information from the followers. Thus, the adjacency matrix of $G$ is denoted by

$$
A=\left[\begin{array}{cc}
0 & 0_{1 \times n} \\
A_{01} & A_{1}
\end{array}\right],
$$

where $A_{1}=\left[a_{i j}\right] \in \mathbb{R}^{n \times n}$ and $A_{01}=\left[a_{i 0}\right] \in \mathbb{R}^{n \times 1}$. Let $G_{2} \triangleq\left(V_{2}, \varepsilon_{2}\right)$ be a fixed and directed graph with $m$ agents. The adjacency matrix of $G_{2}$ is given by $A_{2}=\left[a_{i j}\right] \in \mathbb{R}^{m \times m}$.
The union graph is denoted by $G^{u}=G \cup G_{2}$ with the node set $V^{u}=V \cup V_{2}$ and the edge set $\varepsilon^{u}=\varepsilon \cup \varepsilon_{2}$. Hence

$$
\begin{aligned}
A^{u} & =\left[\begin{array}{ccc}
0 & 0_{1 \times n} & 0_{1 \times m} \\
A_{01} & A_{1} & S_{21}^{\theta(k)} \\
S_{02}^{\theta(k)} & S_{12}^{\theta(k)} & A_{2}
\end{array}\right], \\
A^{\theta(k)} & =\left[\begin{array}{cc}
A_{1} & S_{21}^{\theta(k)} \\
S_{12}^{\theta(k)} & A_{2}
\end{array}\right],
\end{aligned}
$$

where $A^{u}=\left[a_{i j}^{\theta(k)}\right] \in \mathbb{R}^{(n+1+m) \times(n+1+m)}$ is the adjacency matrix of $G^{u}, S_{02}^{\theta(k)} \in \mathbb{R}^{m \times 1}, S_{12}^{\theta(k)} \in \mathbb{R}^{m \times n}$, and $S_{21}^{\theta(k)} \in \mathbb{R}^{n \times m}$ are parts of the switching matrices among the nodes of $G$ and $G_{2}, \theta(k)$ (for brevity, denoted by $\theta_{k}$ ) is a finite homogeneous Markov process, and it will be detailed in the following section.

2.2. Markov Chains. Define a finite set $\Delta=\{1, \ldots, \gamma\}, \gamma \in Z^{+}$, and a set of matrices $S^{\theta_{k}} \in \mathbb{R}^{e \times f}, e, f \in Z^{+}$with the elements $s_{i j}^{\theta_{k}}, i \in[1, e], j \in[1, f]$. There are two sets $\Gamma^{e f}=\Gamma \oplus \Gamma \oplus$ $\cdots \oplus \Gamma$ and $\Gamma_{e f}=\left\{1,2, \ldots, \gamma^{e f}\right\}$, where $\oplus$ represents a novel operation mark among matrices, $\Gamma$ is a set corresponding to $S^{\theta_{k}}$. Meanwhile, introduce the mapping $\Xi: \Gamma^{e f} \rightarrow \Gamma_{e f}$ with

$$
\begin{aligned}
\Xi\left(S^{\theta_{k}}\right)= & s_{11}^{\theta_{k}}+\left(s_{12}^{\theta_{k}}-1\right) \gamma+\cdots+\left(s_{e(f-1)}^{\theta_{k}}-1\right) \gamma^{e f-1} \\
& +\left(s_{e f}^{\theta_{k}}-1\right) \gamma^{e f} .
\end{aligned}
$$

Then, the mapping $\Xi(\cdot)$ is a bijection from $\Gamma^{e f}$ to $\Gamma_{e f}$.

Remark 1. Based on the bijection in (4), the transition probability $\Theta\left(\theta_{k}\right)=\operatorname{Pr}\left[\theta_{k} \mid \theta_{k-1}\right]$ could be derived as follows.

Firstly, for the matrix $S_{12}^{\theta_{k}} \in \mathbb{R}^{m \times n}$, each $\theta_{k} \in \Gamma_{m n}$ is corresponding to the only matrix in the set $\Gamma^{m n}$. In the modes $\theta_{k}$ and $\theta_{k-1}$, the following is yielded:

$$
\begin{aligned}
& S_{12}^{\theta_{k}}=\Xi^{-1}\left(\theta_{k}\right)=\left[\begin{array}{cccc}
s_{11}^{\theta_{k}} & s_{12}^{\theta_{k}} & \cdots & s_{1 n}^{\theta_{k}} \\
s_{21}^{\theta_{k}} & s_{22}^{\theta_{k}} & \cdots & s_{2 n}^{\theta_{k}} \\
\vdots & \vdots & \ddots & \vdots \\
s_{m 1}^{\theta_{k}} & s_{m 2}^{\theta_{k}} & \cdots & s_{m n}^{\theta_{k}}
\end{array}\right] \text {, } \\
& S_{12}^{\theta_{k-1}}=\Xi^{-1}\left(\theta_{k-1}\right)=\left[\begin{array}{cccc}
s_{11}^{\theta_{k-1}} & s_{12}^{\theta_{k-1}} & \cdots & s_{1 n}^{\theta_{k-1}} \\
s_{21}^{\theta_{k-1}} & s_{22}^{\theta_{k-1}} & \cdots & s_{2 n}^{\theta_{k-1}} \\
\vdots & \vdots & \ddots & \vdots \\
s_{m 1}^{\theta_{k-1}} & s_{m 2}^{\theta_{k-1}} & \cdots & s_{m n}^{\theta_{k-1}}
\end{array}\right]
\end{aligned}
$$


Assume that each edge of $G^{u}$ takes value in the set $\Delta$ with an unequal probability. The transition rate matrix is given by

$$
\delta=\left[\begin{array}{cccc}
\delta^{11} & \delta^{12} & \cdots & \delta^{1 \gamma} \\
\delta^{21} & \delta^{22} & \cdots & \delta^{2 \gamma} \\
\vdots & \vdots & \cdots & \vdots \\
\delta^{\gamma 1} & \delta^{\gamma^{2}} & \cdots & \delta^{\gamma \gamma}
\end{array}\right],
$$

where

$$
\begin{array}{rl}
\delta^{x y} \geq 0 & x, y \in \Delta, \\
\sum_{y \in \Delta} \delta^{x y}=1 & x \in \Delta .
\end{array}
$$

In addition, Markov chain is ergodic throughout this paper. It is obvious that

$$
\begin{aligned}
& \operatorname{Pr}\left[\theta_{k} \mid \theta_{k-1}\right]=\operatorname{Pr}\left[\Xi^{-1}\left(\theta_{k}\right) \mid \Xi^{-1}\left(\theta_{k-1}\right)\right] \\
& =\operatorname{Pr}\left[S_{12}^{\theta_{k}} \mid S_{12}^{\theta_{k-1}}\right] \\
& =\operatorname{Pr}\left[\begin{array}{cccc}
\delta\left(s_{11}^{\theta_{k}} \mid s_{11}^{\theta_{k-1}}\right) & \delta\left(s_{12}^{\theta_{k}} \mid s_{12}^{\theta_{k-1}}\right) & \cdots & \delta\left(s_{1 n}^{\theta_{k}} \mid s_{1 n}^{\theta_{k-1}}\right) \\
\delta\left(s_{21}^{\theta_{k}} \mid s_{21}^{\theta_{k-1}}\right) & \delta\left(s_{22}^{\theta_{k}} \mid s_{22}^{\theta_{k-1}}\right) & \cdots & \delta\left(s_{2 n}^{\theta_{k}} \mid s_{2 n}^{\theta_{k-1}}\right) \\
\vdots & \vdots & \ddots & \vdots \\
\delta\left(s_{m 1}^{\theta_{k}} \mid s_{m 1}^{\theta_{k-1}}\right) & \delta\left(s_{m 2}^{\theta_{k}} \mid s_{m 2}^{\theta_{k-1}}\right) & \cdots & \left(\delta s_{m n}^{\theta_{k}} \mid s_{m n}^{\theta_{k-1}}\right)
\end{array}\right] \\
& =\prod_{i=1}^{m} \prod_{j=1}^{n} \delta\left(s_{i j}^{\theta_{k}} \mid s_{i j}^{\theta_{k-1}}\right),
\end{aligned}
$$

where $\delta(\cdot \mid \cdot)$ represents the transition probability from one mode to another. Let the transition probability be $\delta_{i j}^{x y}$ while $s_{i j}^{\theta_{k-1}}=x$, and $s_{i j}^{\theta_{k}}=y$; then

$$
\operatorname{Pr}\left[\theta_{k} \mid \theta_{k-1}\right]=\prod_{i=1}^{m} \prod_{j=1}^{n} \delta_{i j}^{x y} .
$$

The same work is done to the matrices $S_{02}^{\theta_{k}} \in \mathbb{R}^{m \times 1}$ and $S_{21}^{\theta_{k}} \in \mathbb{R}^{n \times m}$. Overall consideration, for brevity, denotes the total probability as

$$
\frac{1}{\omega}=\prod_{i=1}^{m} \prod_{j=1}^{n} \delta_{i j}^{x y}+\prod_{i=1}^{n} \prod_{j=1}^{m} \delta_{i j}^{x y}+\prod_{i=1}^{m} \prod_{j=1}^{1} \delta_{i j}^{x y} .
$$

Finally, the total number of system modes is $\eta=\gamma^{2 m n+m}$, and the transition rate matrix is

$$
\Pi=\frac{1}{\omega} \mathbf{1}_{\eta} \mathbf{1}_{\eta}^{T} .
$$

2.3. PD-Like Consensus Algorithm. Suppose the discrete dynamic of the $i$ th follower is

$$
\xi_{i}(k+1)=\xi_{i}(k)+T u_{i}(k)
$$

where $\xi_{i}(k)$ is the state at $t=k T$, where $k$ is the discrete-time index, $T$ is the sampling period, and $u_{i}(k)$ is the control input.

Let the reference state be $\xi_{0}(k)=\xi^{r}(k)$. Consider the discrete-time coordinated tracking algorithm adopted from [16], together with the Markovian parameter $\theta_{k}$; consensus algorithm (13) will be applied to the agents in graph $G^{u}$ :

$$
\begin{aligned}
& u_{i}(k)=\frac{1}{\sum_{j=0}^{n+m} a_{i j}^{\theta_{k}}} \\
& \quad \cdot \sum_{j=1}^{n+m} a_{i j}^{\theta_{k}}\left[\frac{\xi_{j}(k)-\xi_{j}(k-1)}{T}-q\left(\xi_{i}(k)-\xi_{j}(k)\right)\right] \\
& +\frac{1}{\sum_{j=0}^{n+m} a_{i j}^{\theta_{k}}} \\
& \cdot a_{i 0}^{\theta_{k}}\left[\frac{\xi^{r}(k)-\xi^{r}(k-1)}{T}-q\left(\xi_{i}(k)-\xi^{r}(k)\right)\right],
\end{aligned}
$$

where $a_{i j}^{\theta_{k}}, i=1, \ldots, n+m, j=0, \ldots, n+m$, is the $(i, j)$ th entry of $A^{u}$, and $q$ is a positive constant. Suppose that each follower has at least one neighbor, thus $\sum_{j=1}^{n+m} a_{i j}^{\theta_{k}} \neq 0, i=0, \ldots, n+m$. Appling (12) and (13) yields

$$
\begin{aligned}
& \xi_{i}(k+1)=\xi_{i}(k)+\frac{T}{\sum_{j=0}^{n+m} a_{i j}^{\theta_{k}}} \\
& \quad \cdot \sum_{j=1}^{n+m} a_{i j}^{\theta_{k}}\left[\frac{\xi_{j}(k)-\xi_{j}(k-1)}{T}-q\left(\xi_{i}(k)-\xi_{j}(k)\right)\right] \\
& +\frac{T}{\sum_{j=0}^{n+m} a_{i j}^{\theta_{k}}} \\
& \cdot a_{i 0}^{\theta_{k}}\left[\frac{\xi^{r}(k)-\xi^{r}(k-1)}{T}-q\left(\xi_{i}(k)-\xi^{r}(k)\right)\right] .
\end{aligned}
$$

Define $e_{i}(k)=\xi_{i}(k)-\xi^{r}(k)$; let $E(k)=\left[e_{1}(k), \ldots, e_{m+n}(k)\right]^{T}$ and $\sigma(k)=\left[E^{T}(k+1), E^{T}(k)\right]^{T}$; it follows that

$$
\sigma(k+1)=M^{\theta_{k}} \sigma(k)+N X^{r}(k),
$$

where

$$
\begin{aligned}
& M^{\theta_{k}} \\
& =\left[\begin{array}{cc}
(1-T q) I_{n+m}+(1+T q) D^{-\theta_{k}} A^{\theta_{k}} & -D^{-\theta_{k}} A^{\theta_{k}} \\
I_{n+m} & 0_{(n+m) \times(n+m)}
\end{array}\right], \\
& D^{\theta_{k}}=\operatorname{diag}\left\{\sum_{j=0}^{n+m} a_{1 j}^{\theta_{k}}, \ldots, \sum_{j=0}^{n+m} a_{(n+m) j}^{\theta_{k}}\right\}, \\
& N=\left[\begin{array}{c}
I_{n+m} \\
0_{(n+m) \times(n+m)}
\end{array}\right], \\
& X^{r}(k)=\mathbf{1}_{n+m}\left(2 \xi^{r}(k)-\xi^{r}(k+1)-\xi^{r}(k-1)\right) .
\end{aligned}
$$

$\left\{\sigma(k), k \in Z^{+}\right\}$is not a Markov process, but the joint process $\left\{\sigma(k), \theta_{k}\right\}$ is. Assume that the reference trajectory is a 
deterministic signal instead of a random one. The initial state of the joint process is denoted as $\left\{\sigma_{0}, \theta_{0}\right\}$. It follows that the solution of (15) is

$$
\begin{aligned}
\sigma(k)= & M^{\theta_{k-1}} M^{\theta_{k-2}} \cdots M^{\theta_{0}} \sigma_{0}+N X^{r}(k-1) \\
& +\sum_{l=0}^{k-2} M^{\theta_{k-1}} M^{\theta_{k-2}} \cdots M^{\theta_{l+1}} N X^{r}(l) \\
= & \widehat{M}_{1} \sigma_{0}+N X^{r}(k-1)+\widehat{M}_{2} N X^{r}(l) .
\end{aligned}
$$

Note that the eigenvalues of $\widehat{M}_{1}$ play an important role in the determining of $\sigma(k)$ as $k \rightarrow \infty$.

\section{Convergence Analysis}

Theorem 2. Suppose that the leader has directed paths to all followers 1 to $n+m$ in $G^{u}$, then

$$
\frac{1}{\omega} \sum_{i=1}^{\eta} D^{-\theta_{k}} A^{\theta_{k}}
$$

has all eigenvalues within the unit circle, where $D^{-\theta_{k}}$ is denoted as the inverse of $D^{\theta_{k}}$.

Proof. There exists $D_{12}^{\theta_{k}}$ (resp., $D_{21}^{\theta_{k}}$ ) which is corresponding to $S_{12}^{\theta_{k}}$ (resp., $S_{21}^{\theta_{k}}$ ) as denoted in (16), it follows from (3) that

$$
D^{\theta_{k}}=\left[\begin{array}{cc}
D_{1}+D_{21}^{\theta_{k}} & 0_{n \times m} \\
0_{m \times n} & D_{12}^{\theta_{k}}+D_{2}
\end{array}\right]
$$

Then, it is obvious that

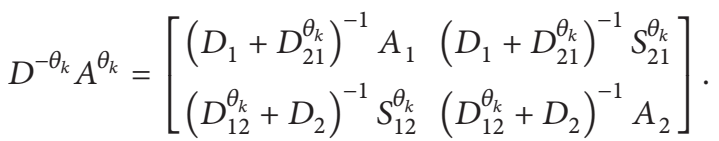

All the elements of $\left(D_{1}+D_{21}^{\theta_{k}}\right)^{-1} A_{1},\left(D_{1}+D_{21}^{\theta_{k}}\right)^{-1} S_{21}^{\theta_{k}},\left(D_{12}^{\theta_{k}}+\right.$ $\left.D_{2}\right)^{-1} S_{12}^{\theta_{k}}$, and $\left(D_{12}^{\theta_{k}}+D_{2}\right)^{-1} A_{2}$ are less than 1 . Based on Lemma 3.1 in [5], (18) has all eigenvalues within the unit circle.

Lemma 3 ([28], Proposition 3.6). Let $L=\left(\Pi^{T} \otimes\right.$ $\left.I_{4 n^{2}}\right) \operatorname{diag}\left\{M^{1} \otimes M^{1}, \ldots, M^{\eta} \otimes M^{\eta}\right\}$ and $\widehat{L}=\left(\Pi^{T} \otimes\right.$ $\left.I_{2 n}\right) \operatorname{diag}\left\{M^{1}, \ldots, M^{\eta}\right\}$, where $M^{i}, i \in[1, \eta]$, is defined in (16); let $\otimes$ represent the Kronecker product of matrices. If $\rho(L)<1$, then $\rho(\widehat{L})<1$, where $\rho(\cdot)$ denotes the matrix spectral radius.

Theorem 4. Suppose that the leader has directed paths to all nodes in the union graph $G^{u}$, while $\tau_{i}^{1}, \tau_{i}^{2}>0$ holds obviously. If the positive scalars $T>0$ and $q>0$ satisfy

$$
T q<\min _{i=1, \ldots, n+m}\left(\tau_{i}^{1}, \tau_{i}^{2}\right)
$$

where

$$
\begin{aligned}
\tau_{i}^{1} & =\frac{1-\left(2 \cos ^{2}(\phi)-1\right) r^{2}-\sqrt{2\left[-6 \cos ^{2}(\phi) r^{2}+3 r^{2}+2 r^{3} \cos ^{3}(\phi)-r^{3} \cos (\phi)-3 r \cos (\phi)-1\right]}}{\left(2 \cos ^{2}(\phi)-1\right) r^{2}-2 r \cos (\phi)+1}, \\
\tau_{i}^{2} & =\frac{1-\left(2 \cos ^{2}(\phi)-1\right) r^{2}+\sqrt{2\left[\left(2 \cos ^{2}(\phi)-1\right) r^{2}-1\right](r \cos (\phi)-1)}}{\left(2 \cos ^{2}(\phi)-1\right) r^{2}-2 r \cos (\phi)+1},
\end{aligned}
$$

then $\widehat{L}$ has all eigenvalues within the unit circle.

Proof.

Step 1. The matrix $A^{u}$ has $\eta$ modes based on the analysis in the Section 2. If the leader has directed paths to all followers, it follows from Theorem 2 that (18) has all eigenvalues within the unit circle. It will be shown that $\rho(\widehat{L})<1$ through the method of perturbation arguments. Hence $\widehat{L}$ can be written as

$$
\begin{aligned}
\widehat{L} & =\left(\Pi^{T} \otimes I_{2 n}\right) \operatorname{diag}\left(M^{1}, \ldots, M^{\eta}\right) \\
& =\frac{1}{\omega}\left[\begin{array}{cccc}
M^{1} & M^{2} & \cdots & M^{\eta} \\
M^{1} & M^{2} & \cdots & M^{\eta} \\
\vdots & \vdots & \ddots & \vdots \\
M^{1} & M^{2} & \cdots & M^{\eta}
\end{array}\right] .
\end{aligned}
$$

Denote the elementary transformation block matrix $P_{1} \epsilon$ $\mathbb{R}^{2 \eta(m+n)^{2} \times 2 \eta(m+n)^{2}}$ as

$$
P_{1}=\left[\begin{array}{cccc}
I_{4(n+m)^{2}} & 0_{2(n+m) \times 2(n+m)} & \cdots & I_{4(n+m)^{2}} \\
0_{2(n+m) \times 2(n+m)} & I_{4(n+m)^{2}} & \cdots & I_{4(n+m)^{2}} \\
\vdots & \vdots & \ddots & \vdots \\
0_{2(n+m) \times 2(n+m)} & 0_{2(n+m) \times 2(n+m)} & \cdots & I_{4(n+m)^{2}}
\end{array}\right] .
$$

The equation can be calculated as

$$
\begin{aligned}
\left|\lambda I_{4 \omega(n+m)^{2}}-\widehat{L}\right| & =\left|P_{1}^{-1}\left(\lambda I_{4 \eta(n+m)^{2}}-\widehat{L}\right) P_{1}\right| \\
& =\lambda^{4(\eta-1)(n+m)^{2}}\left|\lambda I_{4(n+m)^{2}}-\frac{1}{\omega} \sum_{i=1}^{\eta} M^{i}\right| .
\end{aligned}
$$


The issue will be converted to find the conditions to make sure all eigenvalues of $M^{i}$ are within the unit circle.

Step 2. The characteristic polynomial of $M^{i}$ is given by

$$
\begin{aligned}
& \operatorname{det}\left|\lambda I_{2(n+m)}-M^{i}\right| \\
& =\operatorname{det}\left(\left[\begin{array}{rr}
\lambda I_{n+m}-\left[(1-T q) I_{n+m}+(1+T q) D^{-i} A^{i}\right] & D^{-i} A^{i} \\
-I_{n+m} & \lambda I_{n+m}
\end{array}\right]\right) \\
& =\operatorname{det}\left(\left[\lambda^{2}+(T q-1) \lambda\right] I_{n+m}+[1-(1+T q) \lambda] D^{-i} A^{i}\right) .
\end{aligned}
$$

Note that $s_{h}^{i}$ is the $h$ th eigenvalue of $D^{-i} A^{i}$, which is in the unit circle. Define $s_{h}^{i}=r \cos (\phi)+r \sin (\phi) j$, where $r \in[0,1]$ is the length of $s_{h}^{i}, \phi \in[0,2 \pi]$, and $j$ is the imaginary parts signal. Therefore, the roots satisfy

$$
\lambda^{2}+\left[T q-1-(1+T q) s_{h}^{i}\right] \lambda+s_{h}^{i}=0 .
$$

It can be noted that

$$
\begin{aligned}
\lambda_{1}+\lambda_{2}= & -[T q-1-(1+T q) r \cos (\phi)] \\
& +(1+T q) r \sin (\phi) j, \\
\lambda_{1} \lambda_{2}= & r \cos (\phi)+r \sin (\phi) j .
\end{aligned}
$$

Let $\lambda_{1}=l_{1} \cos (\alpha)+l_{1} \sin (\alpha) j$ and $\lambda_{2}=l_{2} \cos (\beta)+l_{2} \sin (\beta) j$. Based on (28), thus

$$
\begin{aligned}
& \sin (\phi)=\sin (\alpha+\beta), \\
& \cos (\phi)=\cos (\alpha+\beta), \\
& r=l_{1} l_{2}, \\
& l_{1} \cos (\alpha)+l_{2} \cos (\beta) \\
& \quad=-[T q-1-(1+T q) r \cos (\phi)], \\
& l_{1} \sin (\alpha)+l_{2} \sin (\beta)=(1+T q) r \sin (\phi) .
\end{aligned}
$$

It follows from (30) that

$$
\begin{aligned}
\left(l_{1} \cos (\alpha)+l_{2} \cos (\beta)\right)^{2}-\left(l_{1} \sin (\alpha)+l_{2} \sin (\beta)\right)^{2} \\
=[T q-1-(1+T q) r \cos (\phi)]^{2} \\
\quad-[(1+T q) r \sin (\phi)]^{2} .
\end{aligned}
$$

Using (29) and (31), after some manipulation, (31) can be rewritten as

$$
\begin{aligned}
2 l_{1}^{2} \cos ^{2}(\alpha)+2 l_{2}^{2} \cos ^{2}(\beta)-\left(l_{1}^{2}+l_{2}^{2}\right) \\
=(1+T q)^{2} r^{2}\left(\cos ^{2}(\phi)-\sin ^{2}(\phi)\right) \\
-2(T q)^{2} r \cos (\phi)+(T q-1)^{2} .
\end{aligned}
$$

Aimed to prove that $\lambda_{1}$ and $\lambda_{2}$ are within the unit circle, with $l_{1} \leq 1, l_{2} \leq 1$, it follows that

$$
-2<2 l_{1}^{2} \cos ^{2}(\alpha)+2 l_{2}^{2} \cos ^{2}(\beta)-\left(l_{1}^{2}+l_{2}^{2}\right)<2 .
$$

Then, the following holds:

$$
\begin{aligned}
(1 & +T q)^{2} r^{2}\left(\cos ^{2}(\phi)-\sin ^{2}(\phi)\right)-2(T q)^{2} r \cos (\phi) \\
& +(T q-1)^{2}+2>0, \\
(1 & +T q)^{2} r^{2}\left(\cos ^{2}(\phi)-\sin ^{2}(\phi)\right)-2(T q)^{2} r \cos (\phi) \\
& +(T q-1)^{2}-2<0 .
\end{aligned}
$$

To get the condition of $T q$, the transition of (34) is made:

$$
\begin{aligned}
g_{1}(T q)= & {\left[\left(2 \cos ^{2}(\phi)-1\right) r^{2}-2 r \cos (\phi)+1\right](T q)^{2} } \\
& +2\left[\left(2 \cos ^{2}(\phi)-1\right) r^{2}-1\right] T q \\
& +\left(2 \cos ^{2}(\phi)-1\right) r^{2}+3>0, \\
g_{2}(T q)= & {\left[\left(2 \cos ^{2}(\phi)-1\right) r^{2}-2 r \cos (\phi)+1\right](T q)^{2} } \\
& +2\left[\left(2 \cos ^{2}(\phi)-1\right) r^{2}-1\right] T q \\
& +\left(2 \cos ^{2}(\phi)-1\right) r^{2}-1<0 .
\end{aligned}
$$

With the limit conditions of $r, \phi$, and (35), the range of $T q$ can be obtained.

Firstly, as is well-known, in the analysis of $g_{1}(T q)$, let

$$
\begin{aligned}
& a=2 \cos ^{2}(\phi) r^{2}-r^{2}-2 r \cos (\phi)+1, \\
& b=2\left[\left(2 \cos ^{2}(\phi)-1\right) r^{2}-1\right]<0, \\
& c=\left(2 \cos ^{2}(\phi)-1\right) r^{2}+3
\end{aligned}
$$

then

$$
\begin{aligned}
& g_{1}(0)=\left(2 \cos ^{2}(\phi)-1\right) r^{2}+3>0, \\
& \Delta_{1}=b^{2}-4 a c=8\left[-6 \cos ^{2}(\phi) r^{2}+3 r^{2}\right. \\
& \left.\quad+2 r^{3} \cos ^{3}(\phi)-r^{3} \cos (\phi)-3 r \cos (\phi)-1\right] .
\end{aligned}
$$

After some manipulation, this yields

$$
T q \in\left[0, \tau_{i}^{1}\right],
$$

where $\tau_{i}^{1}$ satisfies (22) with $\Delta_{1}>0$.

Then, for the condition of $g_{2}(T q)$, the same as $g_{1}(T q)$, it can be obtained that

$$
\begin{aligned}
g_{2}(0) & =\left(2 \cos ^{2}(\phi)-1\right) r^{2}-1<0, \\
\Delta_{2} & =b^{2}-4 a c \\
& =8\left[\left(2 \cos ^{2}(\phi)-1\right) r^{2}-1\right][r \cos (\phi)-1] \\
& >0 .
\end{aligned}
$$

Similarly, we have

$$
T q \in\left[0, \tau_{i}^{2}\right],
$$

where $\tau_{i}^{2}$ satisfies (22) with $a>0$. 
Finally, sufficient condition (21) can be exactly proved. It follows from Lemma 3.1 in [5] that $\left(\sum_{i=1}^{\eta} M^{i}\right) / \omega$ has all eigenvalues within the unit circle. Thus, based on (21), the system tracking errors can be convergent stably.

Remark 5. Markov chains are required to be ergodic; therefore it can be ensured that the leader has directed paths to all followers in $G^{u}$. Certainly, the results can be expanded to the case where all the links are governed by Markov chains; through the mapping in (4), Markovian switching topologies will be addressed finally. From this, large numbers of system modes can be described, and the traditional Markovian switching topologies can be recovered through the adjustment of the links modes and the transition rate matrix. However, it will magnify the calculation load and the unknown or partly unknown transition probability in some scenes will be considered in the future.

Lemma 6 ([16], Lemma 3.5). Let $L$ be defined in Lemma 3. For small enough $\mathrm{Tq}, \rho(L)<1$, if and only if the leader has directed paths to all followers in the union graph rather than the subgraphs.

Theorem 7. Assume that $\xi^{r}(k)$ satisfies the fact that the changing rate is bounded; thus

$$
\left|\frac{\xi^{r}(k)-\xi^{r}(k-1)}{T}\right| \leq \bar{\xi}
$$

the leader has directed paths to all followers 1 to $n+m$ in the union graph. When Theorem 4 holds, using algorithm (13), if there exist $0<\mu<1$ and $\nu \geq 1$, the tracking errors of the agents are ultimately mean-square bounded as follows:

$$
2(m+n) T \bar{\xi} \frac{\nu}{1-\mu} .
$$

Proof. It follows from (15) that

$$
\begin{aligned}
\|\sigma(k)\|_{E}= & \left\|M^{\theta_{k-1}} M^{\theta_{k-2}} \cdots M^{\theta_{0}} \sigma_{0}\right\|_{E} \\
& +\left\|N X^{r}(k-1)\right\|_{E} \\
& +\sum_{l=0}^{k-2}\left\|M^{\theta_{k-1}} M^{\theta_{k-2}} \cdots M^{\theta_{l+1}} N X^{r}(l)\right\|_{E} .
\end{aligned}
$$

Noting that $N X^{r}(k-1)$ is deterministic, based on (41), thus

$$
\left\|N X^{r}(k-1)\right\|_{E} \leq 2 \sqrt{m+n} T \bar{\xi} .
$$

Based on Lemmas 3.4 and 3.5 and Theorem 3.9 in [28], there exist $0<\mu<1$ and $\nu \geq 1$, yielding

$$
\begin{aligned}
& \left\|M^{\theta_{k-1}} M^{\theta_{k-2}} \cdots M^{\theta_{0}} \sigma_{0}\right\|_{E} \leq \sqrt{(m+n) \mu^{2 k} \nu^{2}}\left\|\sigma_{0}\right\|_{2}, \\
& \left\|M^{\theta_{k-1}} M^{\theta_{k-2}} \cdots M^{\theta_{l+1}} N X^{r}(l)\right\|_{E} \\
& \quad \leq 2(m+n) T \bar{\xi} \sqrt{\mu^{2 k-2 l-2} \nu^{2}} .
\end{aligned}
$$

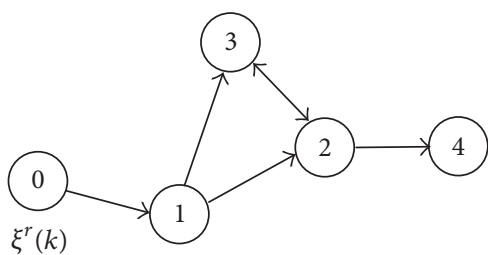

G

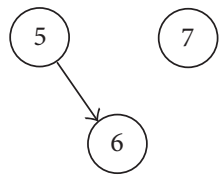

$G_{2}$
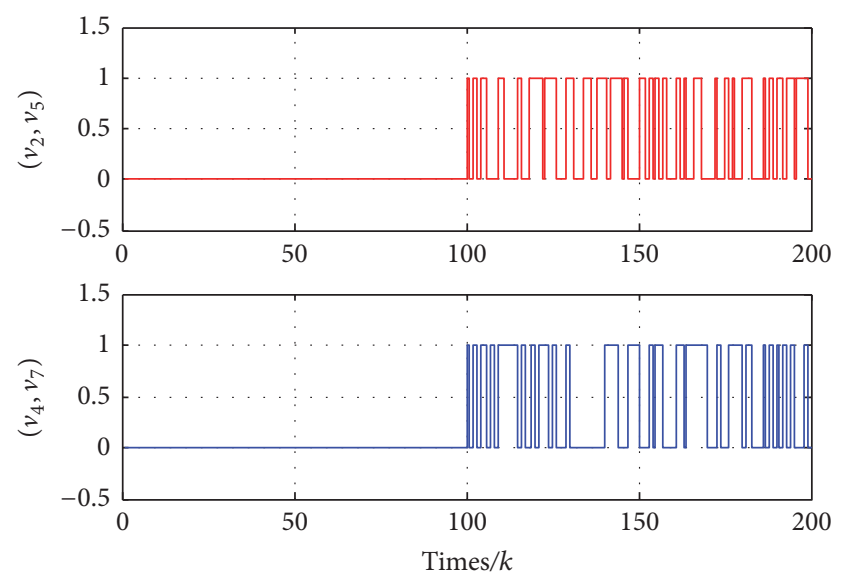

Figure 2: Modes of the edges generated by Markov chains.

Noting that $2 \sqrt{m+n} T \bar{\xi} \leq 2(m+n) T \bar{\xi} \nu$, after some manipulation, thus it follows that

$$
\|\sigma(k)\|_{E} \leq(m+n) \mu^{k} \nu\left\|\sigma_{0}\right\|_{2}+2(m+n) T \bar{\xi} \nu \frac{1-\mu^{k}}{1-\mu} .
$$

Therefore, as $k \rightarrow \infty$, it can be obtained that $\|\sigma(k)\|_{E} \leq 2(m+$ n) $T \bar{\xi} \nu /(1-\mu)$. The same as Theorem 3.2 in [16], the tracking errors will go to zero ultimately as $T \rightarrow 0$. But for the original interaction topology $G$, the ultimate mean-square bound is given by $2 n T \bar{\xi} \nu /(1-\mu)$ through the same method, which is smaller than the union system.

\section{Simulation Results}

In this section, a simulation example is given to verify the effectiveness of the theoretical results. For brevity, let $a_{i j}^{\theta_{k}}=1$ if $\left(v_{j}, v_{i}\right) \in \varepsilon^{u}, i \in[1, n+m]$, and $j \in[0, n+m]$. The subgraphs $G$ and $G_{2}$ are shown in Figure 1 .

It follows from $G^{u}$ that each Markov chain has two modes, which means $\gamma=2$ and $\eta=2^{3 \times 5+3 \times 4}=2^{27}$, and the transition rate matrices are considered as in Table 1 .

As an example, some modes of the edges are shown in Figure 2.

For the PD-like discrete-time consensus algorithm, the initial states of the agents in $G$ and $G_{2}$ are $\left[\begin{array}{llll}\sigma_{1}(0) & \sigma_{2}(0) & \sigma_{3}(0) & \sigma_{4}(0)\end{array}\right]=\left[\begin{array}{llll}3 & 1 & 0 & 2.5\end{array}\right]$ and $\left[\begin{array}{lll}\sigma_{5} & \sigma_{6} & \sigma_{7}\end{array}\right]=\left[\begin{array}{lll}-2 & -3 & -2.5\end{array}\right]$. Furthermore,

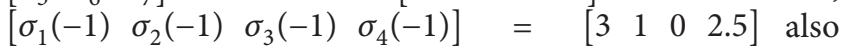


TABLE 1: Transition rate matrices for Markov chains.

\begin{tabular}{|c|c|c|c|c|}
\hline \multirow{2}{*}{$\begin{array}{l}\text { Edges } \\
\text { Transition } \\
\text { rate matrices }\end{array}$} & $\begin{array}{c}\left(v_{1}, v_{5}\right) ;\left(v_{2}, v_{7}\right) ;\left(v_{6}, v_{3}\right) \\
\left(v_{4}, v_{5}\right) ;\left(v_{2}, v_{6}\right)\end{array}$ & $\begin{array}{c}\left(v_{1}, v_{6}\right) ;\left(v_{2}, v_{7}\right) ;\left(v_{3}, v_{6}\right) ; \\
\left(v_{5}, v_{4}\right) ;\left(v_{7}, v_{4}\right) ;\end{array}$ & \multicolumn{2}{|c|}{$\begin{array}{c}\left(v_{1}, v_{7}\right) ;\left(v_{2}, v_{5}\right) ;\left(v_{3}, v_{5}\right) ; \\
\left(v_{4}, v_{6}\right) ;\left(v_{7}, v_{3}\right) ;\end{array}$} \\
\hline & $\delta=\left[\begin{array}{ll}0.3 & 0.7 \\
0.4 & 0.6\end{array}\right]$ & $\delta=\left[\begin{array}{ll}0.2 & 0.8 \\
0.4 & 0.6\end{array}\right.$ & $\delta=$ & {$\left[\begin{array}{ll}0.5 & 0.5 \\
0.8 & 0.2\end{array}\right.$} \\
\hline
\end{tabular}

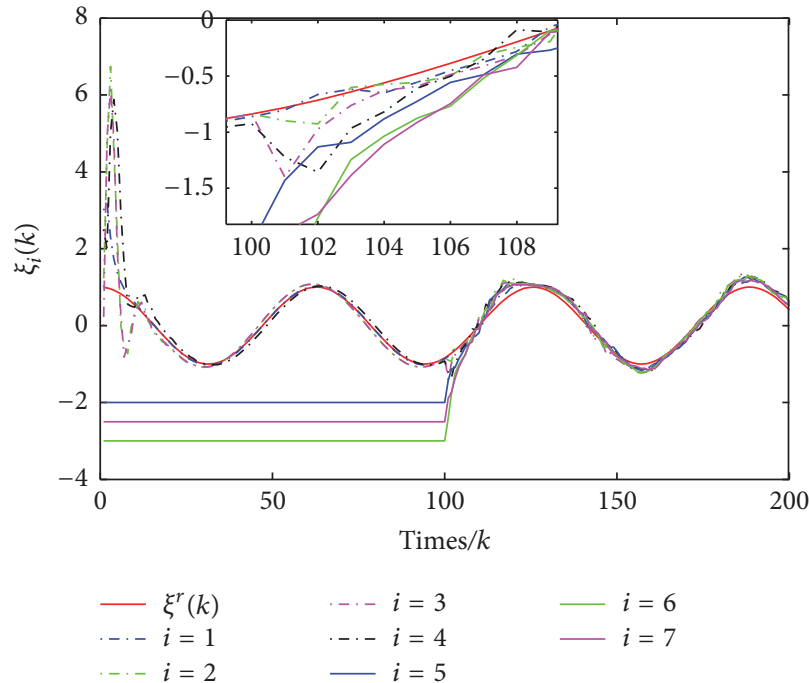

(a) State tracking of all agents

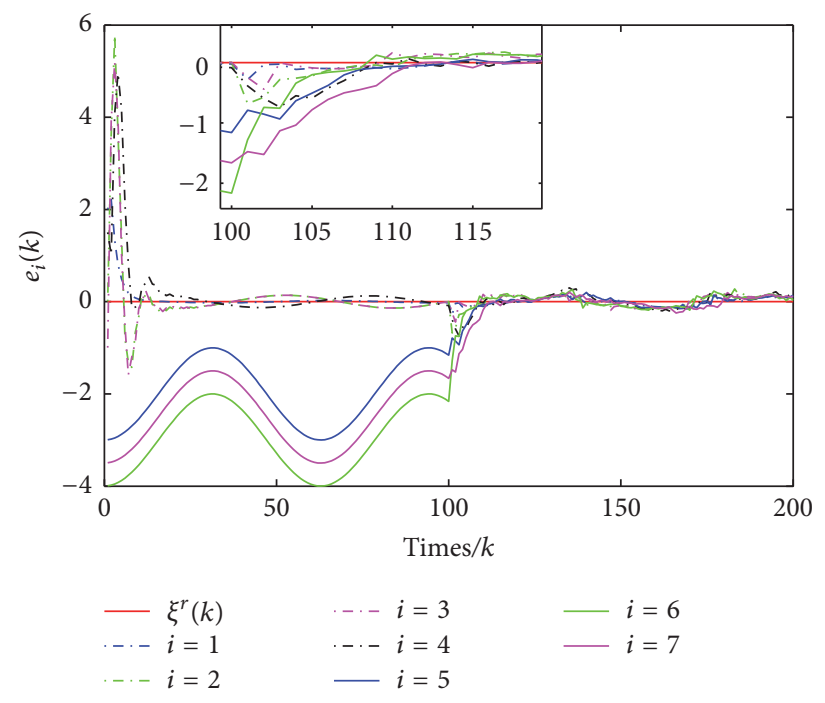

(b) Tracking errors of all agents

FIGURE 3: Simulation results in Case 1.

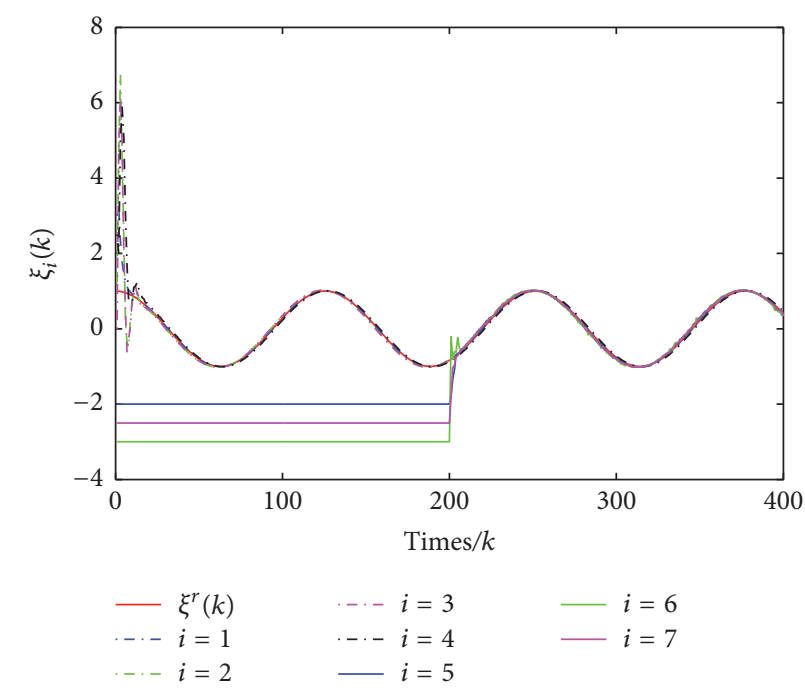

(a) State tracking of all agents

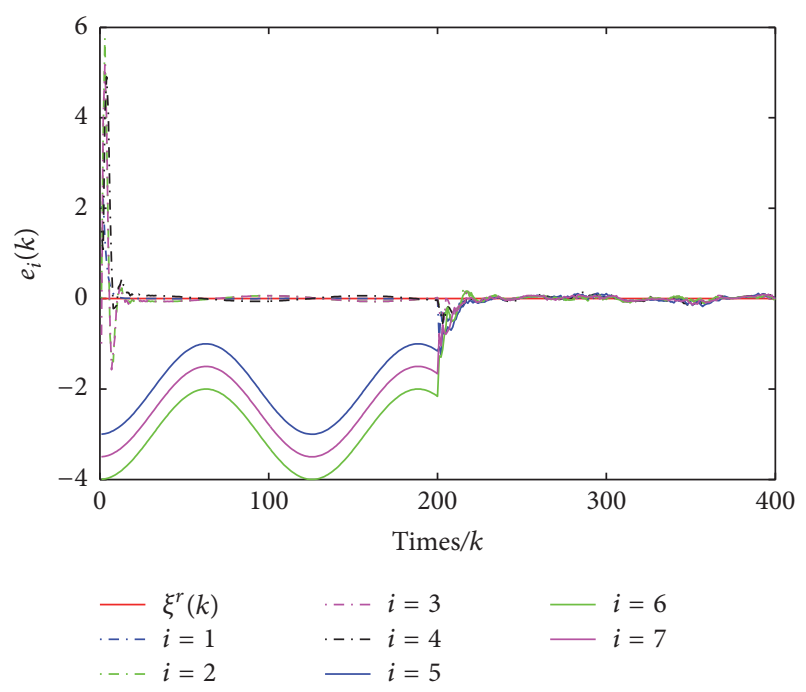

(b) Tracking errors of all agents

FIGURE 4: Simulation results in Case 2.

should be defined at the initial time, and let $\xi^{r}(k)=\cos (k T)$. Distributed controller (13) is implemented with the parameters in the following four cases.

Case 1. $T=0.1, q=4$.

Case 2. $T=0.05, q=4$.
Case 3. $T=0.05, q=2$.

Case 4. $T=0.4, q=2$.

Simulation results are shown in Figures 3-6.

Figure 3 shows the plots of the system states and tracking errors with a time varying reference when $T=0.1, q=4$. 


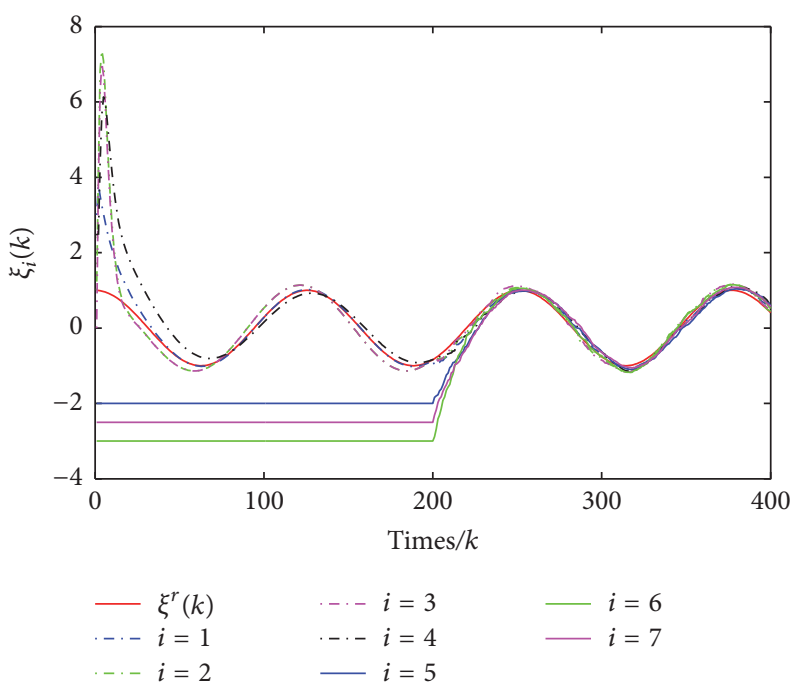

(a) State tracking of all agents

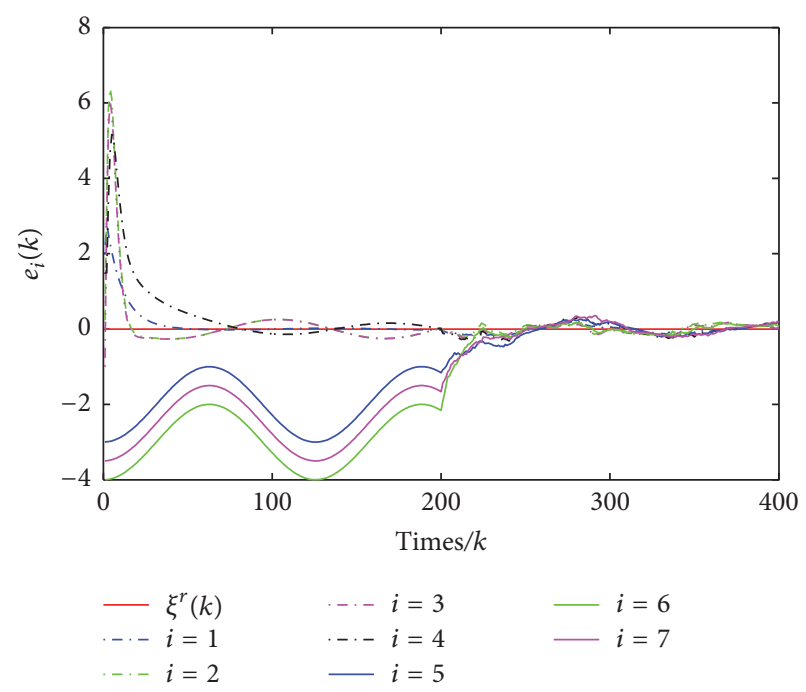

(b) Tracking errors of all agents

FIGURE 5: Simulation results in Case 3.

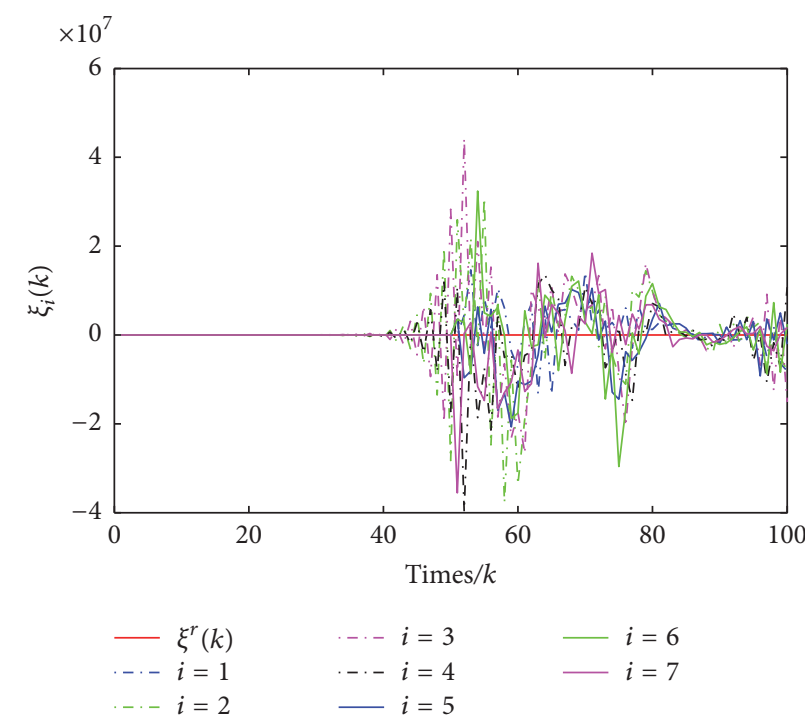

(a) State tracking of all agents

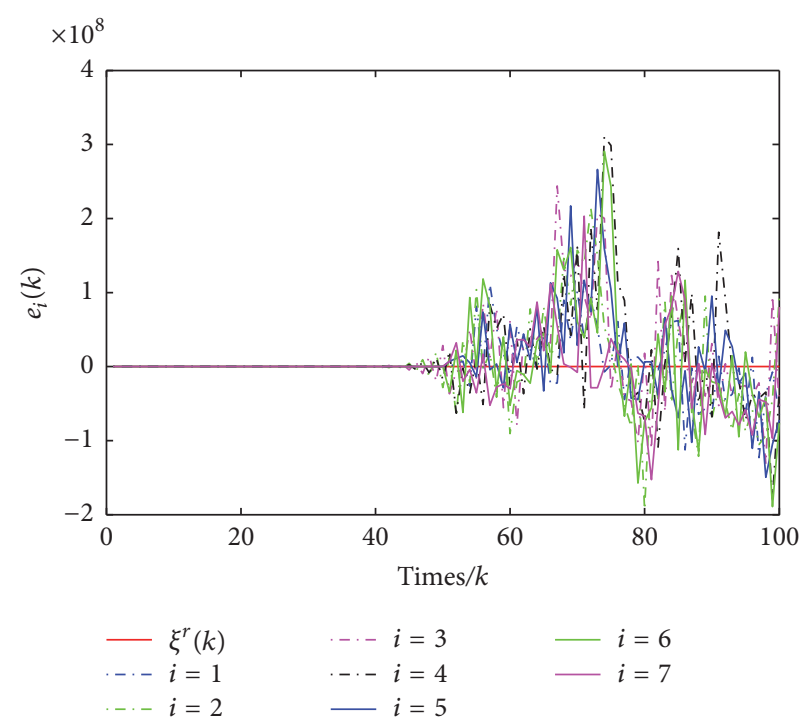

(b) Tracking errors of all agents

Figure 6: Simulation results in Case 4.

Once the two fixed groups are combined at half of the time, all the followers can track the reference finally. More specifically, the system states and tracking errors curves are smooth in the first half of time, but in the rest of time as shown in the partial enlarged details, there are lots of burrs on the plots for all agents obviously, since the links between agents in $G$ and $G_{2}$ are governed by the random Markov chains.

Under the same reference, compared with Figure 3, the system states can track more effectively and the tracking errors are smaller while $T=0.05, q=4$. Furthermore, as shown in Figures 4 and 5, there is a quick response and smaller tracking errors ultimately, along with a bigger control gain $q$ and the same $T$. But for Figure 6 , when $T=0.4, q=2$ in Case 4 , the situation is unpredictable, because $T q$ does not meet the condition of Theorem 4 . It can be noted that the tracking errors become unbounded in this case. What should be stressed more is that, based on Theorem 4, the largest value of $T q$ is approximately equal to 0.44 . Otherwise, a quantitative comparison among the four cases is given in Table 2, which shows the mean and standard deviation of the tracking errors in the second half of the time. The comparison results show that the tracking errors depend on $T$ and $q$ obviously.

\section{Conclusion}

In this paper, distributed discrete-time coordinated tracking control for multiagent systems is investigated to solve the issue on the union graph with Markov chains. Based 
TABLE 2: Comparison results of the tracking errors among the four cases.

\begin{tabular}{lcc}
\hline Cases & Mean & Standard deviation \\
\hline Case 1 & 0.0305 & 0.1718 \\
Case 2 & -0.0163 & 0.1483 \\
Case 3 & -0.0788 & 0.2349 \\
Case 4 & $-4.4958 \times 10^{5}$ & $2.5529 \times 10^{7}$ \\
\hline
\end{tabular}

on a novel mapping, Markovian switching topologies are redesigned through using the Markov chains to the edge set. The PD-like discrete-time consensus algorithm is applied to deal with the time varying reference. A sufficient condition of the match sampling period and a feasible control gain to the time varying reference is obtained in terms of trigonometric function with multiple-term formula. Both the theoretical and simulation results show that the ultimate tracking errors are related to the sampling period. Although we focus on studying the discrete-time multiagent systems with an ideal communication network, an extended analysis may be considered for the case with time delays, which will be addressed in our future work.

\section{Conflicts of Interest}

The authors declare that they have no conflicts of interest.

\section{Acknowledgments}

This work was supported by National Natural Science Foundation of China under Grant 61473248 and Natural Science Foundation of Hebei Province under Grant F2016203496.

\section{References}

[1] J. Liang, Z. Wang, and X. Liu, "Distributed state estimation for discrete-time sensor networks with randomly varying nonlinearities and missing measurements," IEEE Transactions on Neural Networks and Learning Systems, vol. 22, no. 3, pp. 486496, 2011.

[2] X. Dong, Y. Zhou, Z. Ren, and Y. Zhong, "Time-Varying Formation Tracking for Second-Order Multi-Agent Systems Subjected to Switching Topologies With Application to Quadrotor Formation Flying," IEEE Transactions on Industrial Electronics, vol. 64, no. 6, pp. 5014-5024, 2017.

[3] Q. Zhang, P. Li, Z. Yang, and Z. Chen, "Adaptive flocking of non-linear multi-agents systems with uncertain parameters," IET Control Theory \& Applications, vol. 9, no. 3, pp. 351-357, 2015.

[4] T. Liu and Z.-P. Jiang, "Distributed nonlinear control of mobile autonomous multi-agents," Automatica, vol. 50, no. 4, pp. 10751086, 2014.

[5] S. Su and Z. Lin, "Distributed Consensus Control of MultiAgent Systems with Higher Order Agent Dynamics and Dynamically Changing Directed Interaction Topologies," IEEE Transactions on Automatic Control, vol. 61, no. 2, pp. 515-519, 2016.

[6] P. Li and K. Qin, "Distributed robust Ho consensus control for uncertain multiagent systems with state and input delays,"
Mathematical Problems in Engineering, vol. 2017, 15 pages, 2017, ID 5091756.

[7] P. Lin, Y. Jia, and L. Li, "Distributed robust $H \infty$ consensus control in directed networks of agents with time-delay," Systems \& Control Letters, vol. 57, no. 8, pp. 643-653, 2008.

[8] W. He, G. Chen, Q.-L. Han, and F. Qian, "Network-based leader-following consensus of nonlinear multi-agent systems via distributed impulsive control," Information Sciences, vol. 380, pp. 145-158, 2017.

[9] P. Lin, W. Ren, and J. A. Farrell, "Distributed continuous-time optimization: nonuniform gradient gains, finite-time convergence, and convex constraint set," Institute of Electrical and Electronics Engineers Transactions on Automatic Control, vol. 62, no. 5, pp. 2239-2253, 2017.

[10] Y. Cao, W. Ren, and M. Egerstedt, "Distributed containment control with multiple stationary or dynamic leaders in fixed and switching directed networks," Automatica, vol. 48, no. 8, pp. 1586-1597, 2012.

[11] H. Cai and J. Huang, "Leader-following consensus of multiple uncertain Euler-Lagrange systems under switching network topology," International Journal of General Systems, vol. 43, no. 3-4, pp. 294-304, 2014.

[12] Y. Cao, W. Yu, W. Ren, and G. Chen, "An overview of recent progress in the study of distributed multi-agent coordination," IEEE Transactions on Industrial Informatics, vol. 9, no. 1, pp. 427-438, 2013.

[13] Y. Wang and Q. Wu, "Distributed robust Ho consensus for multi-agent systems with nonlinear dynamics and parameter uncertainties," Asian Journal of Control, vol. 17, no. 1, pp. 352361, 2015.

[14] P. Lin, W. Ren, and H. Gao, "Distributed velocity-constrained consensus of discrete-time multi-agent systems with nonconvex constraints, switching topologies, and delays," IEEE Transactions on Automatic Control, vol. 62, no. 11, pp. 5788-5794, 2017.

[15] Y. Su and J. Huang, "Stability of a class of linear switching systems with applications to two consensus problems," Institute of Electrical and Electronics Engineers Transactions on Automatic Control, vol. 57, no. 6, pp. 1420-1430, 2012.

[16] H. Zhao, W. Ren, D. Yuan, and J. Chen, "Distributed discretetime coordinated tracking with Markovian switching topologies," Systems and Control Letters, vol. 61, no. 7, pp. 766-772, 2012.

[17] F. Chen, G. Feng, L. Liu, and W. Ren, "Distributed average tracking of networked Euler-Lagrange systems," Institute of Electrical and Electronics Engineers Transactions on Automatic Control, vol. 60, no. 2, pp. 547-552, 2015.

[18] J. Mei, W. Ren, and G. Ma, "Distributed containment control for Lagrangian networks with parametric uncertainties under a directed graph," Automatica, vol. 48, no. 4, pp. 653-659, 2012.

[19] X. Li, H. Wu, and Y. Yang, "Consensus of heterogeneous multiagent systems with arbitrarily bounded communication delay," Mathematical Problems in Engineering, vol. 2017, Article ID 5679073, 6 pages, 2017.

[20] S. Yu and X. Long, "Finite-time consensus for second-order multi-agent systems with disturbances by integral sliding mode," Automatica, vol. 54, pp. 158-165, 2015.

[21] P. Ming, J. Liu, S. Tan, S. Li, L. Shang, and X. Yu, "Consensus stabilization in stochastic multi-agent systems with Markovian switching topology, noises and delay," Neurocomputing, vol. 200, pp. 1-10, 2016. 
[22] C. Ma, "Finite-time passivity and passification design for markovian jumping systems with mode-dependent timevarying delays," Discrete Dynamics in Nature and Society, vol. 2017, Article ID 3256871, 11 pages, 2017.

[23] D. Xie and Y. Cheng, "Bounded consensus tracking for sampled-data second-order multi-agent systems with fixed and Markovian switching topology," International Journal of Robust and Nonlinear Control, vol. 25, no. 2, pp. 252-268, 2015.

[24] H. Zhao, "Leader-following consensus of data-sampled multiagent systems with stochastic switching topologies," Neurocomputing, vol. 167, pp. 172-178, 2015.

[25] W. Li, L. Xie, and J.-F. Zhang, "Containment control of leaderfollowing multi-agent systems with Markovian switching network topologies and measurement noises," Automatica, vol. 51, pp. 263-267, 2015.

[26] Y. Zhao, G. Guo, and L. Ding, "Guaranteed cost control of mobile sensor networks with Markov switching topologies," ISA Transactions ${ }^{\circledR}$, vol. 58, pp. 206-213, 2015.

[27] X. Liu, X. Yu, and H. Xi, "Finite-time synchronization of neutral complex networks with Markovian switching based on pinning controller," Neurocomputing, vol. 153, pp. 148-158, 2015.

[28] O. L. V. Costa, M. D. Fragoso, and R. P. Marques, Discrete-time Markovian Jump Linear Systems, Springer, London, UK, 2005. 


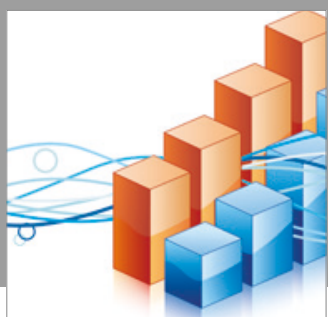

Advances in

Operations Research

vatersals

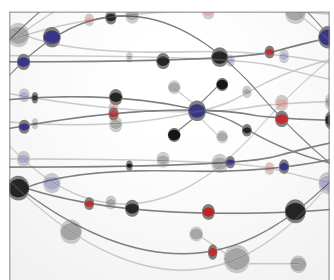

\section{The Scientific} World Journal
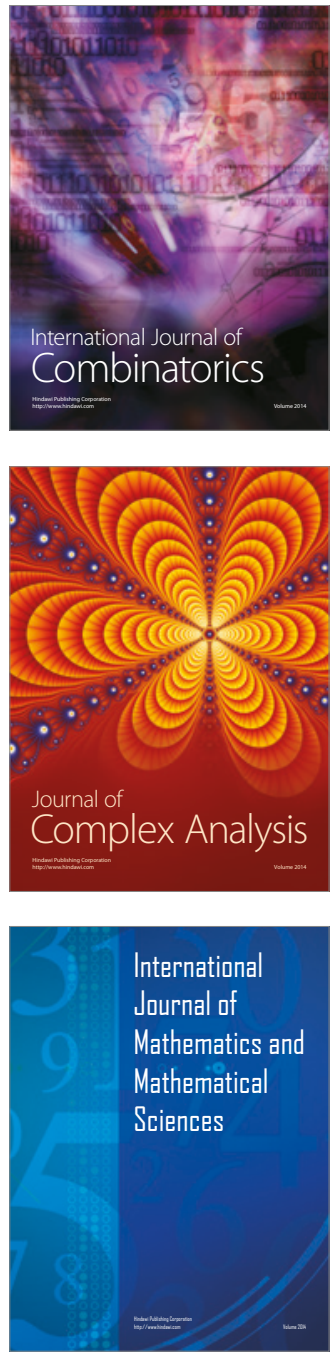
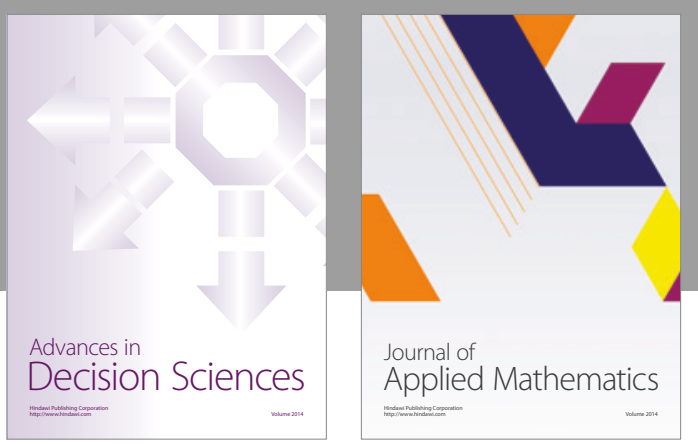

Algebra

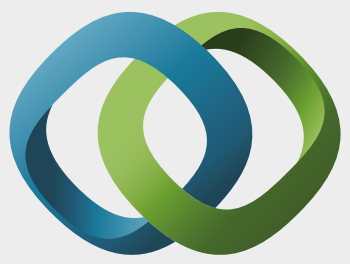

\section{Hindawi}

Submit your manuscripts at

https://www.hindawi.com
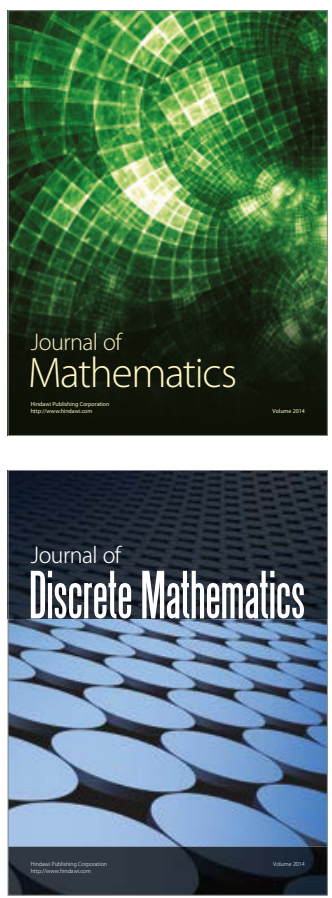

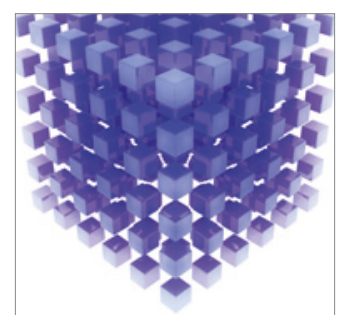

Mathematical Problems in Engineering
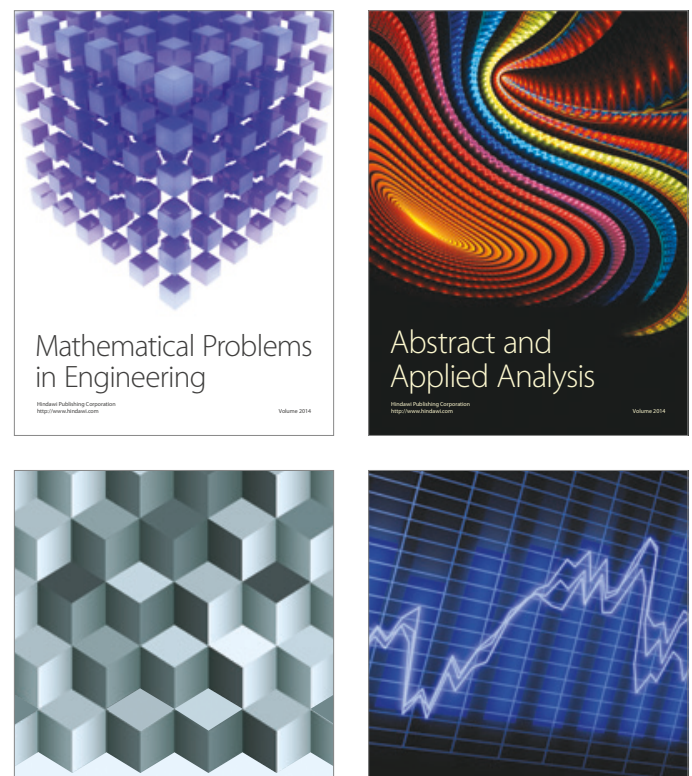

Journal of

Function Spaces

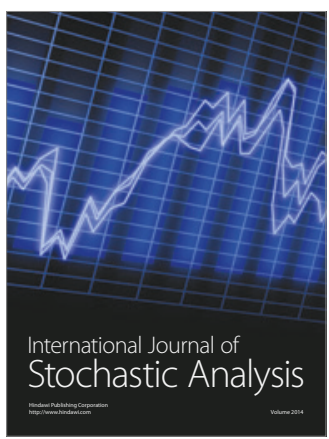

Probability and Statistics
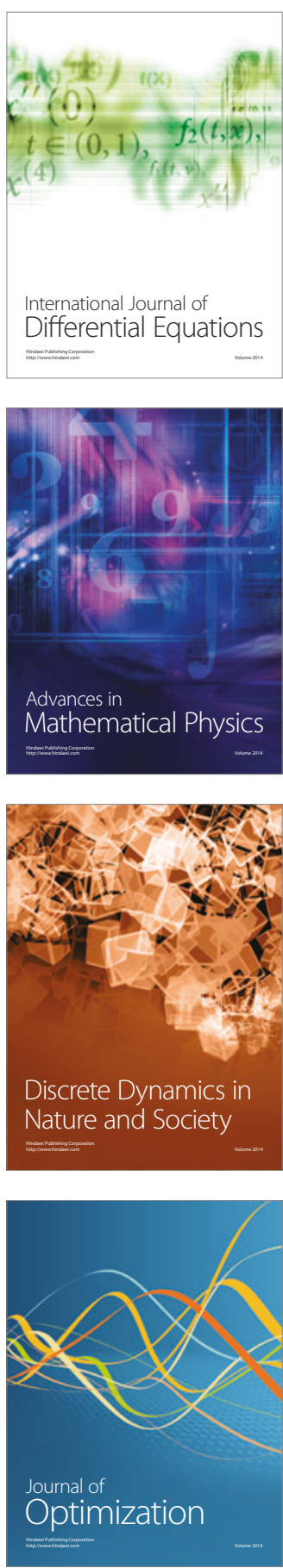